\title{
Gaston Thiesson en Suisse aux côtés de Romain Rolland
}

(septembre 1915-avril 1916)

\section{Roland Roudil}

\section{OpenEdition}

Journals

Édition électronique

URL : http://journals.openedition.org/edl/345

DOI : $10.4000 /$ edl. 345

ISSN : 2296-5084

\section{Éditeur}

Université de Lausanne

\section{Édition imprimée}

Date de publication : 15 septembre 2012

Pagination : 119-142

ISBN : 978-2-940331-28-4

ISSN : 0014-2026

\section{Référence électronique}

Roland Roudil, « Gaston Thiesson en Suisse aux côtés de Romain Rolland », Études de lettres [En ligne], 3 | 2012, mis en ligne le 15 septembre 2015, consulté le 19 décembre 2020. URL : http:// journals.openedition.org/edl/345; DOI : https://doi.org/10.4000/edl.345 


\section{GASTON THIESSON EN SUISSE AUX CÔTÉS DE ROMAIN ROLLAND (SEPTEMBRE 1915-AVRIL 1916)}

Romain Rolland et Gaston Thiesson se rencontrent en août 1912, mais leur amitié se renforce lors des deux séjours du peintre en Suisse durant la Première Guerre mondiale, entre septembre 1915 et avril 1916. La venue de Thiesson, au moment où Rolland décide de se retirer de la mêlée, est un réconfort pour l'écrivain attaqué par la presse nationaliste qui l'accuse de défaitisme. Il voit en lui un homme courageux et dévoué qui veut recueillir des témoignages de soutien à paraître dans Les Hommes du Jour. Après son installation au bord du lac Léman, Thiesson séjourne à Montana, avant un voyage à travers la Suisse. Puis il rentre en France où il meurt trois ans plus tard. Cette correspondance révèle la volonté exigeante de Rolland de mettre en accord une pensée, une vie et un art pour mieux les opposer tous ensemble aux forces destructrices de la haine, dans la recherche héroïque entre les êtres et les peuples.

Dans la vie de Romain Rolland, la figure du peintre Gaston Thiesson est étroitement liée à la Suisse puisqu'il vint par trois fois rendre visite à l'écrivain dans ce pays: d'abord à Schönbrunn en août 1912, puis trois ans plus tard pendant la guerre: en 1915, du 8 au 18 septembre, avant un séjour prolongé avec sa femme d'octobre de la même année à avril 1916.

Ces deux derniers séjours voient l'amitié de Romain Rolland et de Gaston Thiesson se construire et s'enraciner dans la réalité concrète de l'action. Dans leur correspondance, en partie inédite, sur les 225 lettres échangées, 86 - soit plus du tiers - sont rédigées lors de la présence du peintre en Suisse, pays qui se révèle être le lieu privilégié d'une collaboration très active entre les deux hommes ${ }^{1}$.

I. Voir les correspondances de R. Rolland avec sa mère (R. Rolland, Je commence à devenir dangereux, abrégé $C 20$ ), J.-R. Bloch (Deux hommes se rencontrent, abrégé $C 5$ ), A. de Châteaubriant (L'Un et l'Autre II, abrégé C 30), le Journal des années de guerre 
Attaché à la Suisse, le nom du peintre est également associé au premier conflit mondial et aux épreuves vécues par l'écrivain depuis la déclaration de guerre. Thiesson, réformé du service militaire pour raison de santé, rejoint Rolland qui échappe à la mobilisation par son âge. Ces quelques mois qu'un auteur désormais célèbre et un peintre de seize ans son cadet passent ensemble dans cet observatoire qu'est la Suisse à cette époque méritent attention: l'arrivée de Thiesson à Vevey a lieu à un moment clé de la vie de l'écrivain. Les attaques contre lui, en cette année 1915, se faisant de plus en plus virulentes, il décide en effet d'interrompre son activité journalistique et songe à privilégier les relations avec ceux qui en France ou à l'étranger commencent à le soutenir ou à défendre des idées proches des siennes. Le peintre Thiesson fait partie de ceux-là.

\section{Rencontre à Schönbrunn}

Gaston Thiesson, qui est loin de jouir à cette époque de la même notoriété que Rolland, a épousé en 1908 Renée Boulet, la fille du drapier Emmanuel Boulet, propriétaire d'une manufacture de textile à Elbeuf et dont la fille aînée, Denise, est l'épouse du célèbre couturier parisien et grand collectionneur d'œuvres d'art Paul Poiret. Dans le catalogue des œuvres de sa collection, dont la vente aux enchères publiques eut lieu en 1925 à l'Hôtel Drouot, des tableaux de Thiesson, se trouvent en bonne place aux côtés de ceux de Van Dongen, Matisse, Picabia, Utrillo ou Vlaminck. Le biographe de Paul Poiret note que le couturier éprouva pour son beau-frère autant d'admiration que d'amitié ${ }^{2}$. Grâce à la fortune de son épouse et de son beau-père, le peintre a sans doute obtenu les subsides lui permettant d'assurer les frais de ses visites en Suisse chez Rolland, en 1912 et en 1915-1916. La correspondance fait certes allusion à des ventes d'œuvres auprès de galeries suisses, mais elles n'ont sans doute pas suffi à financer de tels déplacements.

Le critique d'art Christophe Rameix le présente comme un peintre impressionniste de l'Ecole de Crozant qui subit l'influence de Sisley,

(abrégé $J A G$ ) et P. J. Jouve, Romain Rolland vivant. Sauf indications, les lettres citées sont inédites (Bibliothèque nationale de France, fonds Romain Rolland).

2. P. White, Poiret le magnifique, p. 96. 
Pissarro et Cézanne, certes sans grande prétention inventive mais possédant néanmoins sa propre personnalité:

Il est le petit dernier, qu'on cajole pour son allure d'étudiant bohème. Barbe en bataille et cheveux longs, insouciant du lendemain, il introduit à Crozant un petit air montmartrois. A ses aînés, il inspire plus de tendresse que de jalousie ${ }^{3}$.

La correspondance nous apprend quels sont ses thèmes de prédilection, l'artiste peignant à l'huile sur toile des monuments religieux (Le parvis de l'église, 1905; Eglise en Normandie, 1908), des paysages marins (Pêcheurs à Saint Tropez, 1905), des portraits, comme celui d'Andrée Jouve, du poète Charles Vildrac $(1913)^{4}$ ou d'enfants 5 .

Rolland et Thiesson font connaissance par l'intermédiaire de JeanRichard Bloch, fondateur de la revue L'Effort (devenue L'Effort libre en mars 1912). Avec Assouline, Thiesson en est l'illustrateur. Dans cette revue bimensuelle de littérature et d'art, il publie également des articles sur la peinture ${ }^{6}$. Pendant la guerre, le peintre se souviendra des rédacteurs de L'Effort libre et puisera dans ce groupe d'amis de même sensibilité le nom de ceux qui seront susceptibles de venir à la rescousse de l'auteur de Jean-Christophe.

Une première rencontre a lieu en août 1912 alors que Romain Rolland réside à Menzingen-Schönbrunn, près du lac des Quatre Cantons. Le peintre, arrivant à l'improviste, lui apporte des dessins, lui montre ses tableaux. Aussitôt rentré en France, après ce séjour éclair de trois jours, il lui écrit une lettre chaleureuse:

3. Ch. Rameix, L'Ecole de Crozant, p. 109.

4. Portrait exposé à la bibliothèque Georges Duhamel de Mantes-la-Jolie, lors d'une rétrospective C. Vildrac, 17-28 février 1979.

5. The Burlington Magazine for Connoisseurs, vol. 41, 235 (oct. 1922), p. 188-193.

6. "Notes sur la peinture» (juillet et octobre 1910), "La ligne et la couleur» (décembre 1910), "L'homme de goût» (octobre 1911), "Réflexions sur l’Art» (mai-juin 1912), «Notes sur Cézanne» (novembre 1912), "La vie artistique et littéraire» (février 1914), "La vie artistique, le Salon des Indépendants et les critiques» (avril 1914). 
Depuis deux jours, je me promène dans Paris et je rêve. Je ne puis croire la réalité. Je vous ai vu. Je vous connais. Je suis pleinement heureux, trop heureux. Je vous ai dit que je vous aimais, croyez-le ${ }^{7}$.

Le jour même où Thiesson lui écrit cette lettre enthousiaste, Romain Rolland écrit à Jean-Richard Bloch pour lui exprimer, suite à cette courte visite qui l'a «surpris et touché», l'attachement qu'il éprouve pour le peintre:

Quel être intelligent et vivant, plein de générosité! C'est une belle nature; elle m'est très sympathique ${ }^{8}$.

Placées sous le signe de l'art, les premières lettres ${ }^{9}$ de Thiesson expriment une attente de lumière. Le peintre lui offre un amour humble et fort, de nature filiale, tout prêt à s'emplir de la sérénité, de la droiture de son maître, de son amour pour l'humanité souffrante, de sa maîtrise de soi - lui si impulsif:

Avec mon père vous êtes l'homme que j'aime et admire le plus dans le monde. Oui, monsieur Romain Rolland, acceptez mon amitié. Vous êtes bon. Vous êtes fort. Je vous écoute. Toutes les paroles que vous prononcez, je les recueille pieusement. Je les répète avec amour ${ }^{10}$.

Les propos sur l'art en général et plus précisément sur les effets de la technique picturale dans l'émergence de l'émotion esthétique forment ainsi le contenu des premières lettres de cette correspondance ${ }^{11}$.

En octobre 1912, Rolland quitte la Suisse pour Paris où il restera jusqu'au mois d'avril de l'année suivante. Comme il vient d'accepter la proposition d'Edmond Rossier de tenir une chronique dans sa revue

7. Lettre du 9 août 1912, in A. Youssofi (éd.), Correspondance entre Romain Rolland et Gaston Thiesson, p. 25.

8. Lettre du 9 août 1912, C 15, p. 126.

9. Cette correspondance d'avant-guerre de 59 lettres a été étudiée par A. Youssofi (éd.), Correspondance entre Romain Rolland et Gaston Thiesson.

Io. Ibid.

II. Les plus révélatrices des conceptions esthétiques de R. Rolland ont paru dans La Revue française: "Lettres sur l'Art par Romain Rolland», 25 mai 1948, p. 17-20 (6 lettres). 
La Bibliothèque universelle ${ }^{12}$, il s'informe des derniers mouvements de la création picturale, et découvre la peinture contemporaine. A Paris, il visite les musées, se rend dans les collections privées. Au Salon d'automne, il retrouve Thiesson aux yeux de qui rien ne saurait égaler ces moments de rencontre, ni les échanges sur la peinture, ni la correspondance. Chez le collectionneur Vollard, Rolland apprécie, dans les portraits de Cézanne "les mêmes qualités de peintre" ${ }^{13}$ que celles mises en œuvre dans ses paysages. Puis ce sont des échanges de bons procédés entre chroniqueurs de l'art. "J'ai beaucoup aimé votre article sur Cézanne» écrit Rolland à Thiesson qui vient de publier un article sur le peintre ${ }^{14}$. "J'aime votre article sur l'art parisien. Quand et où pourrai-je me procurer ceux qui suivront?» ${ }^{15}$ écrit Thiesson qui vient de lire son papier sur le «rythme de l'évolution de la peinture française, depuis Eugène Delacroix" ${ }^{16}$. Le dialogue s'instaure par articles interposés: Rolland, qui apprécie en Thiesson le critique d'art, a découvert Cézanne.

Dans ses lettres au peintre, il manifeste sa foi en la suprématie de l'âme et du "génie du cœur" sur la technique de l'art pour l'art, précise son idée de l'art populaire, exprime son point de vue sur la «nature dominée par l'esprit souverain. Tout est dans l'équilibre des deux termes ${ }^{17}$. Cependant, en bon peintre pragmatique, Thiesson est plus à l'aise dans l'acte créateur que dans la théorisation, dans la vie concrète partagée que dans le maniement des concepts. Réaliser le portrait de son ami reste ainsi le moyen privilégié de concilier son point de vue avec celui de Rolland tout en se situant sur le plan de l'échange artistique. Lors de son bref séjour à Schönbrunn, il esquisse deux dessins de Rolland, que celui-ci envoie à Bloch pour qu'ils illustrent un numéro de L'Effort que le rédacteur veut lui consacrer ${ }^{18}$. Lors de leur rencontre

I2. S. Stelling-Michaud, «Romain Rolland, Edmond Rossier et la Bibliothèque Universelle».

I3. R. Rolland, De Jean-Christophe à Colas Breugnon, p. 55 et 61.

I4. Lettre du 14 novembre 1912, in A. Youssofi (éd.), Correspondance entre Romain Rolland et Gaston Thiesson, p. 55. Il s'agit de l'article intitulé «Notes sur Cézanne», paru dans L'Effort libre, p. 603-608.

I5. Lettre du 6 décembre 1912, in A. Youssofi (éd.), Correspondance entre Romain Rolland et Gaston Thiesson, p. 62.

I6. "Chronique parisienne», Bibliothèque universelle, 205 (janv. 1913), p. 169-184.

I7. Lettre du 29 octobre 1912, in A. Youssofi (éd.), Correspondance entre Romain Rolland et Gaston Thiesson, p. 47.

I8. Ce numéro ne paraîtra pas. 
à Paris, Thiesson prend quelques croquis de lui. Si les photos le rendent présent, rien ne vaut le portrait où transparaît sa grandeur d'âme. Mais le modèle se refuse de poser et le peintre se voit contraint de travailler à partir de croquis ou de clichés photographiques. Rolland, qui ne peut admettre poser des heures durant, ne s'associera jamais à ce projet. Le 23 février 1917, deux ans avant sa mort, le peintre lui écrit encore: "Je ne désespère pas de faire votre portrait, ami insaisissable. ${ }^{19}$.

Le 12 avril 1915, dans l'espoir d'obtenir des nouvelles d'un soldat disparu, Thiesson reprend contact en adressant une lettre à l'Agence internationale de prisonniers de guerre de Genève où travaille son ami. Jusqu'à leur prochaine rencontre en septembre, 28 lettres sont ainsi échangées dont 16 de la plume du peintre, beaucoup plus longues et révélant une affection enthousiaste, fébrile et spontanée. Juste avant son départ pour Genève, il lui écrit: "Je suis un peu fatigué. Ça va me faire du bien d'aller en Suisse. ${ }^{20}$. En effet: son implication auprès de l'écrivain est allée crescendo. Il s'est démené pour se procurer les articles de Rolland, les recopier et les envoyer à ses amis afin de sensibiliser et activer ces «réseaux de sociabilité intellectuelle» constitués avant la guerre autour de ces deux hommes qui lui sont proches: Jean-Richard Bloch et les rédacteurs de L'Effort libre, Charles Vildrac et les amis de L'Abbaye. Il a obtenu l'adresse de Rolland par l'intermédiaire d'Albert Doyen qui s'était associé en 1907 au groupe de Créteil et lié d'amitié avec Vildrac et Duhamel. Aussitôt le peintre donne à Rolland des nouvelles des uns et des autres: Bazalgette, Chennevières, J.-R. Bloch - mais il sait que Rolland est en rapport étroit avec lui - Jules Romains, Duhamel, puis Bachelin, Durtain, Mercereau et Arcos.

Jusqu'à mi-juillet, Thiesson n'est pas tout entier impliqué dans sa mission, mais deux événements vont donner du sens à son existence et lui faire prendre la direction de la Suisse: la découverte des attaques virulentes de Henri Massis contre le "défaitiste" Romain Rolland ${ }^{21}$ et la mort du lieutenant Farise, cet officier tué sur le front, professeur à

I9. Lettre du 23 février 1917.

2o. Lettre du $1^{\text {er }}$ septembre 1915.

2I. Le 7 juillet avait paru chez Floury Romain Rolland contre la France de Henri Massis (JAG, p. 443 sq.). Cette brochure comprenait deux articles: l'un paru le 30 août 1913 dans l'Opinion, intitulé "Romain Rolland ou le dilettantisme de la foi", repris en 1924 dans Jugements II, Paris, Plon, p. 139-156; l'autre dans l'Opinion du 24 avril 1915, «Romain Rolland parle». 
l'institut de Saint-Pétersbourg. Il ne lui est plus possible de rester inactif devant «le meurtre des élites".

De son côté, Rolland le met au courant de ce qu'il sait du conflit et des forces qui s'organisent en Europe pour dénoncer cette sauvage barbarie. Son point de vue, pense-t-il, est la somme des visions que chacun des journaux européens lui rapporte quotidiennement, du champ de bataille aux manœuvres diplomatiques, des agitations gouvernementales aux activités économiques de guerre. Aussi résume-t-il dans ces premières lettres la responsabilité des pays belligérants avant d'affirmer que «c'est une époque formidable» ${ }^{22}$.

Il est vrai que la grande offensive journalistique de Loyson n'a pas encore été lancée. Cet homme, qui offre l'image d'un homme pétri de bons sentiments, est le fils de Charles Loyson (1827-1912), prédicateur plus connu sous son nom religieux de Père Hyacinthe, et excommunié pour avoir remis en cause le dogme de l'infaillibilité pontificale. Le fils Paul-Hyacinthe, né à Genève en 1873, est dramaturge, mais c'est surtout un polémiste prolixe et redoutable, profondément pacifiste avant la guerre, républicain, tolstoïen et admirateur de Jean-Christophe. Mais en août 1914, son pacifisme a fait long feu. Comme beaucoup, il s'est rallié à l'idéologie de l'«union sacrée» et ne comprend pas l'attitude de Rolland dont il deviendra, en cette année 1915, l'adversaire le plus dangereux.

Quand Thiesson reprend contact, tous les articles qui figureront plus tard dans le recueil Au-dessus de la mêlée ont été publiés, hormis «Le Meurtre des Elites», "Littérature de Guerre» et "Jaurès» que Rolland fait parvenir au peintre dès parution. Il lui en envoie d'autres: "Les Idoles", «Inter arma caritas", "De deux mots le moindre» et finit par lui demander, avant son arrivée en Suisse: "Avez-vous lu mes deux articles du Journal de Genève "Pour l'Europe", parus en janvier et février?»"23. Thiesson a fait preuve d'initiative dans ses activités de documentation et a montré du courage dans sa "Protestation" parue dans La Guerre Sociale du 15 juillet ${ }^{24}$ contre la brochure de Massis.

Les deux hommes se rapprochent: leurs lettres débutent toutes par "cher ami», "mon cher ami», avec, de la part du peintre, une expression plus affectueuse dans sa lettre du 8 août: "mon très cher ami ".

22. Lettre du 19 avril 1915.

23. Lettre du 2 septembre 1915.

24. Lettre du 16 juillet 1915. 
Leur correspondance se concentre sur les sujets de polémique dans lesquels Rolland est entraîné et porte sur l'élaboration par Thiesson d'un ensemble de témoignages de soutien en faveur de Rolland face aux assauts répétés de Loyson et Charles Albert, ainsi que sur les prises de position de trois journaux parisiens: Les Hommes du Jour, Le Bonnet rouge, et la Guerre Sociale. Quand le peintre s'apprête à rejoindre son ami, celui-ci a quitté l'Agence internationale des prisonniers de guerre. Dans l'Internationale Rundchau de Zurich, une lettre de lui a paru fin juillet dans laquelle il déclarait: "Je sens l'inutilité de persister davantage. ${ }^{25}$. Début août, il a publié "Jaurès», son dernier article au Journal de Genève. C'est alors que Thiesson s'engage à ses côtés dans la bataille: au moment où Romain Rolland, lui, a décidé de s'en retirer.

\section{A La Tour-de-Peilz}

A Thoune depuis le $1^{\text {er }}$ août, Rolland se rend à Vevey et s'installe le 7 septembre à l'hôtel Mooser. Le lendemain Thiesson arrive en Suisse. Avant de quitter la France, il a chargé Jacques Mesnil de s'occuper des témoignages. Lors de ce premier séjour, Thiesson apporte à l'écrivain des nouvelles des quelques esprits français qui lui sont restés fidèles et rend compte du dévouement des amis de Paris avec lesquels, sur les conseils de Rolland, il s'est mis en relation. Le peintre a-t-il été à la hauteur de la tâche?

En tous cas, il s'est déjà fait remarquer par les autorités douanières qui lui ont confisqué une lettre fermée de Georges Pioch destinée à l'écrivain. Or il était interdit de faire passer du courrier à un destinataire autrement que par voie postale aux termes d'une loi qui avait pour but de protéger la Poste de la concurrence, en réalité, par ces temps de guerre, pour obliger toute correspondance au contrôle de la censure. L'incident fera l'objet d'une information dans la presse nationale, d'un article polémique de Souday dans le Temps et d'une contravention à l'encontre du peintre. Révélant au grand jour un lien qui se voulait discret entre «le pacifiste de Genève» et un journal non conformiste de Paris, Les Hommes du Jour, la confiscation de la lettre est peut-être à l'origine

25. Reproduit dans Le Journal de Genève, 31 juillet 1915, sous le titre: «Une lettre de Romain Rolland». 
de l'accusation ultérieure d'insoumission dont le peintre sera l'objet dès son retour en France. Rolland n'avait pas besoin d'une telle publicité.

Après quatre journées passées auprès de son "maître", qui resteront marquées dans sa mémoire, Thiesson se rend à Zurich, y rencontre René Arcos, puis se rend à Berne avant de retrouver Rolland à Vevey et de regagner Genève le lendemain où il rend visite à Guilbeaux avec qui il passe deux soirées. Le rédacteur de la revue Demain pense grand bien de lui :

J'apprécie sa franche et chaude cordialité et cette naïveté qu'il possède encore et qu'ont perdu $[s i c]$ de bonne heure tous les littéraires. Nous avons passé ensemble deux longues et délicieuses soirées et vous pensez bien qu'il y fut presque exclusivement question de vous. Son idée de témoignage est excellente ${ }^{26}$.

La visite de Thiesson au consulat constitue le moment important de son passage à Genève, puisqu'il est question de l'«affaire» de la lettre fermée: Reboul, une connaissance de Rolland, l'introduit auprès du viceconsul et vise lui-même son passeport. Il semble que ce soit l'écrivain qui ait conseillé au peintre la marche à suivre pour se tirer de ce mauvais pas. Lors de la condamnation de Thiesson par le parquet de la Seine ${ }^{27}$, Rolland offrira de se charger de payer l'amende.

Thiesson est de retour en France le 18 septembre. Il écrit à Rolland de Paris le jour même: il se montre indigné par un article de Loyson intitulé «Lettre à Marie Milliet». Plus tard, il apprend à son correspondant qu'il a rédigé une réplique à Charles Albert à paraître sous peu dans Les Hommes du Jour. Thiesson poursuit son combat militant en faveur de Rolland, qui à propos de cet article, écrira: "C'est franc et brave, comme vous êtes. ${ }^{28}$. Animé du même zèle, il se met en rapport avec le peintre Matisse dont le frère est porté disparu sur le front et se rend chez Humblot pour suivre de près la publication de la brochure d'articles. De son côté, l'écrivain cherche à le mettre en contact avec Gustave Dupin, dont La Guerre infernale paraitra dans la revue de Guilbeaux, lui communique son adresse, celles de Martin du Gard et du peintre Simon Bussy. Le 3 octobre, Rolland écrit à sa mère:

26. Lettre du 17 septembre 1915.

27. $J A G$, p. 622.

28. Lettre du 6 octobre 1915. 
Lettre débordante d'affection de Thiesson. Il répète le «J'ai un ami» de Christophe. Il est actuellement en Bretagne et pense revenir en Suisse dans une quinzaine ${ }^{29}$.

En attendant, il envoie au peintre des articles de presse, lui conseille la lecture de journaux, le tient au courant de la parution de son recueil chez Ollendorff et de ses démêlés avec la censure. Le "brave Thiesson" se démène: il confie à Rolland qu'il est fatigué et qu'il a hâte de venir en Suisse pour s'y reposer avec sa femme et, revenu de Bretagne à Paris, exprime le désir de s'installer à Port-Courbet. Avant son départ, il rend visite aux parents de Rolland à Paris, et en route, va voir son ami Bloch, hospitalisé à Lyon. "Depuis deux mois, j’ai connu beaucoup de gens nouveaux», confie-t-il, avant d'ajouter: «trop ${ }^{30}$.

Pour ce second séjour, Thiesson arrive avec sa femme le 11 octobre à Genève. Cette fois-ci, tout s'est bien passé à la frontière. Par contre, à la gare, le couple n'a pu récupérer qu'une bicyclette, l'autre ayant été égarée $^{31}$. Thiesson remet à Rolland la copie des témoignages d'intellectuels qui se sont prononcés en sa faveur et qui vont être publiés dans Les Hommes du Jour et prend connaissance de la note que le peintre a rédigée pour accompagner la publication.

Secondé par les journalistes Mesnil, Pioch et Desprès, qui continueront sa tâche maintenant qu'il a quitté la France, le peintre ne limite plus son rôle à celui de militant. C'est aussi le passionné d'art qui vient rendre visite à son ami à qui il prête, spécialement apporté de France, l'ouvrage de Georges Riat sur Courbet. Dans une lettre à sa sœur, Rolland évoque longuement l'arrivée des Thiesson avec qui l'écrivain a dîné la veille au soir :

Je n'ai pas besoin de te dire qu'il [Gaston Thiesson] a passé la soirée entière à causer sans être parvenu, je crois, à minuit et quart, à achever la phrase qu'il avait commencée à 7 heures.

Puis il ajoute:

29. C 20, p. 170.

30. Lettre du 8 octobre 1915.

31. Lettre à Madeleine Rolland, 6 octobre 1915. 
Je dis minuit et quart... Il a fallu que le voisin (un peu Brésilien) donnât des coups de pied désespérés dans la muraille pour qu'il se décidât à aller rejoindre sa compagne ${ }^{32}$.

Les époux Thiesson s'installent dans une pension à La Tour-de-Peilz pour y prendre du repos. "J'ai l'intention de consacrer une huitaine à Thiesson, et il est probable que je ferai quelques promenades avec lui", écrit Rolland à sa sœur ${ }^{33}$. L'écrivain s'installe à Vevey. Pendant une dizaine de jours, les deux hommes ne s'écrivent plus mais se téléphonent, se rencontrent, se promènent. La collecte des témoignages se poursuit:

Cet ami dévoué regrette de ne plus être à Paris. Pour un peu, il y retournerait afin de presser les choses, en ma faveur. Il trouve tous mes autres amis trop mous, trop lents, trop indifférents ${ }^{34}$.

Le 22 octobre, deux jours avant de retourner à Genève, Rolland note dans son Journal:

Promenade avec Thiesson et sa femme à Vernayaz, Saint-Maurice-Bex, par une journée d'automne fraîche et magique, où sur l'ombre bleue des pentes vêtues de feuillages roux et sur les vallées voilées, le chaud soleil illumine les cimes dorées. Comme un rêve d'art resplendissant et lointain dans la brume de la vie, avec un fidèle ami à mes côtés ${ }^{35}$.

La veille de son départ, Rolland reçoit un coup de téléphone de Humblot, le directeur des éditions Ollendorff: il a obtenu le visa définitif de la censure pour son volume d'articles qui portera le titre de $A u$-dessus de la mêlée. Thiesson raccompagne son ami à Genève. Le 27 octobre, Rolland note: "chères paroles affectueuses de Thiesson, de retour à La Tour-de-Peilz, après 15 jours passés ensemble» ${ }^{36}$. Le jour même, il écrit à sa mère sa lassitude. Ces quelques jours passés avec le peintre l'ont apparemment fatigué:

32. Lettre du 12 octobre 1915.

33. Ibid.

34. Lettre à Madeleine Rolland, 19 octobre 1915.

35. $J A G$, p. 556.

36. $J A G$, p. 558. 
Je regrette souvent de n'être pas malade, pour avoir le droit de m'enfermer dans un de ces couvents médicaux des Hautes-Alpes [...]. Mais je n'en ai pas le droit - non seulement parce que beaucoup d'êtres plus faibles ont besoin de moi et de mon exemple, - mais parce que j’ai du moins une poignée d'amis fidèles. Un Thiesson est vraiment d'un dévouement à toute épreuve. Vous ne sauriez trop l'aimer. Il n'y a pas une pensée en lui qui soit occupée de lui-même. Il se donne tout entier $^{37}$.

Certes, Thiesson est «un cœur d'or», mais Rolland manifeste des réticences vis-à-vis de l'action de ses amis. De plus, même s'il s'est tu depuis son article "Jaurès" au Journal de Genève, ses ennemis ne désarment pas. Il vient de prendre connaissance de cet article de Loyson, paru le 15 août dans La Revue, que Thiesson lui a transmis et dans lequel Rolland est accusé d'avoir tenu des propos à une certaine Marie Milliet en faveur de l'Allemagne: «aucun ami n'a eu le courage de me l'envoyer, et il a fallu que ce fût Thiesson qui me le donnât, ces jours-ci", écrit-il à sa mère ${ }^{38}$. A la fin du mois d'octobre, au moment où il apprend que Thiesson va probablement partir pour Montreux et la haute montagne, Rolland reprend son travail à l'Agence internationale des prisonniers de guerre où sa collaboration se poursuit vraisemblablement jusqu'à la fin de l'année ${ }^{39}$.

A l'instant même où paraît Au-dessus de la mêlée, Rolland se montre plus réservé dans son engagement «contre la haine»: la guerre perd à ses yeux son caractère politique, prend «de plus en plus l'aspect d'une révolution de la nature ou d'un fléau religieux infligé à l'Europe. Nul moyen d'en finir que quand les deux camps seront épuisés" ${ }^{40}$.

La lecture de la correspondance de Rolland avec les membres de sa famille permet de relativiser l'importance que revêt pour lui la publication des témoignages. Il a d'ailleurs la joie à cette époque de faire la rencontre de Charles Baudouin, le rédacteur du Carmel, et Thiesson lui annonce l'arrivée de Jouve à Vevey, accompagné de sa femme Andrée et de son fils Olivier. Le poète, avec lequel Rolland va bientôt se lier d'amitié, s'installe même dans un chalet non loin de celui de Thiesson ${ }^{41}$

37. Lettre du 27 octobre 1915, C 20, p. 193.

38. Lettre du 31 octobre 1915, C 20, p. 187.

39. B. Duchatelet, Romain Rolland tel qu'en lui-même, p. 188.

40. Lettre à Madeleine Rolland, 30 octobre 1915.

4I. P. J. Jouve, Romain Rolland vivant, p. 27. 
et le 4 novembre, Rolland se rend à Vevey pour rendre visite à ses amis ${ }^{42}$. Très touché par le peintre qui lui apporte son soutien sans ménager ses peines, il continue à lui exprimer son affection: "Je bénis le sort qui m’a donné un ami comme vous.» ${ }^{43}$. Les épanchements affectifs de Thiesson le poussent à exprimer le fond de son âme:

Vous avez pris une grande place dans mon cœur. Pour vous dire la vérité, votre pensée, la pensée que vous vivez est des très rares qui m'aident à vivre ${ }^{44}$.

Se laissant aller aux confidences, sans doute pour entrer en empathie avec son correspondant, il lui fait part de ses moments de doute: "L'homme constamment m'a déçu.» Redevable du soutien que lui apporte le peintre, il se dit disposé à lui venir en aide s'il a des ennuis, lui conseille la prudence:

Tenez-moi au courant de votre vie, et ne faites pas d'imprudences. Allez piano... ${ }^{45}$. En un mois et demi, les deux hommes ont échangé pas moins de trente-deux lettres.

Les Thiesson et les Jouve quittent La Tour-de-Peilz et s'installent audessus de Montana le 7 novembre. Les amis restent en contact: «Reçu lettres d'installation à Montana " ${ }^{46}$, écrit Rolland à sa mère deux jours plus tard et à Bloch: "Nous avons souvent parlé de vous, Thiesson, Jouve et moi et $[. .$.$] nous vous aimons bien. { }^{47}$. De son côté, le peintre, qui veut faire venir son ami, l'informe qu'il a trouvé pour lui un hôtel confortable entre sa maison et celle de Jouve, l'Hôtel du Grand Chalet. Il lit une nouvelle fois "Le Buisson Ardent", relit le courrier que lui envoie Rolland et affiche même un portrait de lui dans sa chambre. Il est le sommet d'une montagne vers lequel, en bon marcheur, il grimpe. Mais derrière ces amitiés affichées des dissensions apparaissent: Bloch se plaint de ce que son attitude dans cette guerre ne soit pas comprise par le peintre:

\footnotetext{
42. $J A G$, p. 574.

43. Lettre du 26 octobre 1915.

44. Lettre du 2 novembre 1915.

45. Lettre du 5 novembre 1915.

46. C 20, p. 207.

47. Lettre du 11 novembre 1915, C 15, p. 325.
} 
Ne me reproche-t-il pas, par lettre, de verser dans l'égoïsme, de négliger la compréhension et l'amour d'autrui? Zèle de néophyte, qui m'a fait sourire, avec amitié ${ }^{48}$.

Georges Pioch, qui vient de prendre connaissance de la brochure de Guilbeaux, intitulée Pour Romain Rolland, n'apprécie pas de lire qu'il a des «idées flottantes ${ }^{49}$. Rolland voit ses amis se brouiller entre eux. Une suite de propos déformés qui font de Rolland un propagandiste du défaitisme auprès des poilus du front, occupe une grande partie de la correspondance. Rolland résume cette affaire dans une lettre à sa sœur Madeleine:

[...] Thiesson [...] m’a écrit 3 lettres en un jour - tour à tour accablé, rasséréné, puis de nouveau accablé, puis rasséréné derechef... Zut pour toute la tribu des nerveux - dont je suis! - Au bout du compte, tout le monde a déformé les choses, à commencer par Guilbeaux, à continuer par Copeau puis par Thiesson, puis Debarges et finalement par moi. Et le mieux est de déclarer l'affaire close ${ }^{50}$.

Quant aux témoignages, avant même qu'ils soient publiés, le projet est ébruité. Certes, ils finissent par paraitre dans Les Hommes du Jour à partir du 27 novembre ${ }^{51}$, mais il s'agit moins de lettres de soutien que d'une simple «enquête». D'ailleurs le journal cesse de paraître le $1^{\text {er }}$ janvier et les témoignages y seront distillés plus tard au compte-gouttes avant la disparition définitive du quotidien.

Même si la parution de $A u$-dessus de la mêlée chez Ollendorff est un motif de satisfaction, Rolland rappelle à Guilbeaux son projet depuis le mois de juillet "de ne plus écrire d'articles politiques ou sociaux» et que "[son] travail personnel maintenant est tout artistique» ${ }^{52}$. Alors qu'il se tourne vers l'étude de Shakespeare et qu'il pense pouvoir répondre favorablement à une demande d'invitation de la British Academy pour

48. Lettre du 19 novembre 1915, C 15, p. 327.

49. Lettre à R. Rolland, 18 décembre 1915. Voir aussi la lettre du 22 décembre de R. Rolland à G. Thiesson.

5o. Lettre du $1^{\text {er }}$ décembre 1915. Sur cette "affaire», voir C. Sicard (éd.), Correspondance Jacques Copeau-Roger Martin du Gard, p. 797-801.

5I. Les autres témoignages ont été publiés dans les numéros du 4, 11, 18 et 25 décembre 1915, 1 $1^{\text {er }}$ janvier, 11, 18 et 25 mars, 6 et 13 mai 1916.

52. B. Duchatelet, Romain Rolland tel qu'en lui-même, p. 187. 
une conférence sur le dramaturge anglais, des événements continuent néanmoins de le préoccuper.

L'attribution du Prix Nobel par l'Académie suédoise relance ainsi la polémique autour de son nom. Les réactions ne se font pas attendre: «Encore M. Romain Rolland», écrit Le Cri de Paris le 5 décembre 1915. P.-H. Loyson se manifeste de nouveau dans son article «Appel à Romain Rolland " ${ }^{3}$. «Emu par la nouvelle qu'il se prépare un mouvement de sympathie en [sa] faveur (pétition de Thiesson, Mesnil etc.) ", Loyson lui tend la main à condition qu'il se renie, puisque le journaliste, l'ayant soupçonné d'adhérer à une ligue allemande, le Neues Bund Vaterland, l'accuse de trahison ${ }^{54}$. Fidèle à sa ligne de conduite, Rolland ne réplique pas, mais Thiesson va le faire à sa place ${ }^{55}$ de manière bien gauche d'ailleurs, ce qui va attirer l'attention de la presse sur son ami. Sous le feu des attaques, fermement décidé à ne pas répondre, Rolland aimerait que ses amis le fassent à sa place, mais quand ils le font, il juge leurs interventions maladroites.

Quant à Thiesson, déçu par le demi-échec de la publication des témoignages, il s'éloigne maintenant de l'activité journalistique. Il est venu en Suisse pour rencontrer son maître, lui donner les preuves de son amitié, le défendre contre les calomnies de la presse nationaliste. Mais maintenant, songe-t-il, il doit se remettre à sa peinture, et pour créer, comme pour préserver sa santé, des moments de solitude s'imposent. Or il finit par confier à Rolland qu'il est gêné par la présence de Jouve. Voyant l'état d'esprit des Jouve et des Thiesson se détériorer, Rolland ne peut s'empêcher de donner des conseils à ses cadets:

Cher ami, vous vous tourmentez, et j'ai peur que tous deux, Jouve et vous, vous ne mettiez en commun vos soucis, vos tristesses. Je crois, un bon remède, c'est, quand on a une propension au pessimisme et en même temps une vie ardente, de se mettre courageusement en face de la pire hypothèse, de la bien regarder, de se dire: "Quand cela serait!...» Et puis, de se tourner vers toutes les belles et bonnes choses de la vie: la nature, l'art, l'amitié, le génie. Elles n'en sont que meilleures ${ }^{56}$.

53. La Revue, $1^{\mathrm{er}}-15$ novembre 1915, repris par Le Temps, 10 novembre: "Le cas Romain Rolland".

54. JAG, p. 571-589.

55. Lettre au Directeur du Cri de Paris, Les Hommes du Jour, 4 décembre 1915.

56. Lettre du 9 novembre 1915. 


\section{A Montana}

La correspondance change maintenant de tonalité. Thiesson évoque ses excursions, ses activités de peintre et sa relation avec Jouve. Pour Rolland les deux hommes sont bien insouciants dans leurs promenades. Et jouant parfaitement son rôle de conseiller, il écrit sur un ton paternel:

On ne joue pas avec la montagne. Il faut aller doucement, apprendre à la connaître et à l'apprivoiser. [...] Il ne faut pas vouloir manger le Mt Blanc à votre petit déjeuner, la Jungfrau au dîner et le Mt Rose au souper ${ }^{57}$.

Et deux jours plus tard, s'adressant à Thiesson, mais songeant sans doute aussi à Jouve:

Ne vous fatiguez pas trop, cher ami. Habituez votre tête à cet air glacé. S'il se produisait quelque indisposition prolongée (maux de tête, insomnies, diarrhée), redescendez à Sierre pour quelques jours, et réacclimatez-vous ensuite, progressivement, à la hauteur ${ }^{58}$.

Depuis qu'il partage son quotidien avec les Jouve, Thiesson se sent frustré de ne plus avoir une relation privilégiée avec Rolland. Il ne peut trouver un moment favorable pour lui écrire longuement, comme il avait coutume de le faire jusqu'alors. Il en éprouve du dépit: soit Rolland leur écrit à tous deux et il se voit confondu avec Jouve; soit il a une relation singulière avec le poète et le peintre se voit exclu de leur amitié.

En attendant l'arrivée souhaitée de Rolland, les deux hommes font des promenades dans la montagne: Thiesson se dirige vers le Mont Lachaux pour aller peindre, mais les mauvaises conditions météorologiques l'en empêchent. Le temps passe et le peintre n'a toujours pas touché ses pinceaux:

Ce matin je me suis promené avec Jouve qui s'est très bien trouvé de cette promenade dans la neige. [...] Le paysage est féerique, mais adieu le Mont Lachaux ${ }^{59}$.

57. Ibid.

58. Lettre du 11 novembre 1915.

59. Ibid. 
Parfois les deux amis descendent jusqu'à Montana. Thiesson décrit une chapelle qui l'a beaucoup étonné, et fait penser à la chapelle de Brigue décrite dans Le Monde désert ${ }^{60}$ :

Dehors, à droite de la porte sur le mur, il y a une immense croix de bois, ayant la forme d'une épée plantée en terre! Oui, c'est bien ça: dans les mains des hommes ce symbole de la bonté et du sacrifice est devenu celui de la discorde et du meurtre ${ }^{61}$.

Quand, Jouve "n'est pas très bien " ${ }^{62}$ et qu'il ne peut sortir, Thiesson exprime le désir de faire de la peinture, brosser par exemple le portrait d'une paysanne vêtue du costume national du Valais. Un autre jour, il se propose d'aller peindre un paysage, mais alors qu'il a installé son chevalet, le vent se met à souffler et le brouillard commence à voiler les montagnes, ce qui l'oblige à regagner le chalet. Jouve et Thiesson font parfois du ski, ce qui crée de la bonne humeur entre les deux hommes:

Je l'aperçus avec ses skis sous ses bras. Quand il me vit dans mon accoutrement, il éclata de rire. J'avais absolument l'air de faire partie d'une expédition Scott! Nous remontâmes ensemble. Et quand nous eûmes atteint le sommet de la colline, je me débarrassai de mon bagage et nous fîmes du ski. Jouve qui n'avait pas encore essayé de ce sport, fit de merveilleuses glissades sur le derrière ${ }^{63}$.

Descente à ski, soirée près de la cheminée, arrosée de Chianti, lecture d'articles, telles sont les activités que rapportent Thiesson dans ses lettres illustrées de croquis. Leur destinataire s'en montre enchanté:

J'ai reçu votre lettre avec le croquis, dont j'ai bien ri. Je suppose que c'est Jouve qui lève les pieds au ciel, et que c'est vous qui descendez fièrement, les pieds en bas, avec deux harpons à la main, ou deux foudres de Jupiter. - Si vous voulez entremêler vos propos de croquis, langage parlant et langage parlé, j’en serai ravi ${ }^{64}$.

6o. P. J. Jouve, Le monde désert, p. 171 sq.

6I. Lettre du 14 novembre 1915.

62. Expression utilisée dans cette même lettre.

63. Lettre du 15 novembre 1915.

64. Lettre du 24 novembre 1915. 
Parfois les Thiesson invitent chez eux les Jouve qu' ils voient journellement, descendent à Sierre, y déjeunent au Grand Hôtel, où les attend un déjeuner offert par Gallimard ${ }^{65}$ et arrosé de Chianti et de Malvoisie. Mais la neige continue de tomber et empêche dorénavant les promenades. Quant à Rolland, il lit Shakespeare en vue d'écrire ses articles, et correspond un peu moins avec Thiesson...

Le rapport de l'activité quotidienne du peintre forme ainsi le contenu de la plupart des lettres qu'il adresse à Rolland. Ne pouvant travailler à l'extérieur du fait du mauvais temps, il entreprend maintenant les portraits des Jouve et des Gallimard. Pierre Jean a bien du mal à se libérer, n'honore pas toujours les rendez-vous fixés par le peintre ou bien arrive en retard:

[...] après la séance Jouve, je me suis senti fatigué. Il pose peu de temps et assez mal. Comme je le laisse parler il faut que je l'écoute en même temps que je peins. Si je lui impose le silence, il ferme les yeux ou se plaint! 66

Le soir, Jouve leur lit Pascal. Dans ses lettres à Rolland, Thiesson fait le point sur sa technique picturale. Ses toiles s'éclairent, prennent vie. Tout lui paraît neuf: le monde, les couleurs et les sentiments. Il observe ce qui le différencie de son ami Jouve, qui se montre plus désabusé que lui. Finalement des dissensions se font jour. Fidèle à son maître Tolstoï, négateur de l'autorité, Jouve exprime des conceptions bien éloignées de celles du peintre, elles-mêmes plus proches de celles de Rolland lorsqu'il affirmait sa croyance en une force cosmique:

Jouve me parle constamment de la Liberté, du libre arbitre. C'est un sujet indiscutable comme tous les grands sujets. Naturellement je suis contre lui. Pauvre libre arbitre, pauvre cerveau, pauvre volonté, pauvre moucheron! ${ }^{67}$

Et il ajoute plus loin, précisant sa position par rapport à celle du poète:

65. Lors de la Première Guerre mondiale, Gaston Gallimard cherche par tous les moyens à se faire réformer et fait plusieurs séjours dans des sanatoriums, notamment en Suisse. Voir P. Assouline, Gaston Gallimard, p. 103-119.

66. Lettre du 24 novembre 1915.

67. Lettre du 10 novembre 1915. 
Jouve aurait besoin de vivre chez des paysans et d'abandonner ses idées avec ses vêtements de citadin. Nous discutons quelquefois. Nous ne sommes pas souvent d'accord. Je suis exaspéré d'entendre ces mots: Devoir, conscience, bien des hommes. Zut! Il ne faut pas être plus Tolstoyen que Tolstoy, mais essayer d'être pleinement ce qu'on doit être ${ }^{68}$.

Les amis de Montana ont le plaisir de lire un compte-rendu du livre de P. J. Jouve, Vous êtes des Hommes, dans le Journal de Genève du 16 décembre par Paul Seippel, auteur de la première biographie de Romain Rolland. Il est le premier à voir qu'autour de Rolland se constitue un groupe d'amis venus l'aider dans sa tâche. A la fin décembre, Thiesson fait la connaissance de deux artistes, Jacques Lenoir et Edmond Bille, qui serviront de modèle à Jouve dans Le Monde désert: Jacques le Todi et Siemens ${ }^{69}$.

Mais le séjour de Thiesson à Montana se termine. De retour à Genève, où il restera pendant six semaines, il loue pour un mois un appartement dans le quartier des Eaux Vives, dans une maison "moderne", écrit Rolland, mais bon marché avec chauffage central et chambre de bain ${ }^{70}$. Il s'est lié d'amitié avec Edmond Bille, et comme il a l'intention de travailler, il a retenu un modèle. Il a en outre rencontré le peintre Hodler dans son atelier. Son attitude auprès de Rolland a sensiblement changé. Sans doute a-t-il été déçu que son ami n'ait pas voulu le rejoindre à Montana, alors qu'il était avec Jouve.

Durant le mois de janvier, les Thiesson rendent plusieurs fois visite à Rolland ${ }^{71}$, ennuyé de voir son ami toujours accompagné de sa femme. Le peintre n'a plus la relation d'intimité qu'il avait avec lui à l'automne précédent, surtout lors de son premier séjour de septembre. Les lettres de Rolland à sa mère le montrent très critique sur le travail de Thiesson. Il lui reproche de vouloir publier des «tâtonnages»: «C'est comme si, moi,

68. Lettre du 10 novembre 1915.

69. Sur Jacques le Todi, voir J. P. Jouve, En miroir-Journal sans date, p. 48. Edmond Bille (1878-1931), peintre suisse, graveur, et maître-verrier, a créé les vitraux autour de l'autel de la cathédrale de Lausanne. Voir aussi P. J. Jouve, Romain Rolland vivant, p. 33.

70. Lettre du 13 janvier 1916, C 20, p. 212. Jouve habite exactement 25 rue Merle d'Aubigné. Une carte de R. Rolland est adressée à G. Thiesson le 27 janvier 1916 au 23 rue Merle d'Aubigné.

7I. Voir les lettres de Rolland à sa mère des 13, 18 et 24 janvier 1916, C 20, p. 212, 217 et 223. 
je publiais mes brouillons.» Rolland a refusé de se faire photographier et met en doute les dispositions de Thiesson à produire des dessins :

On dirait qu'il ne peut faire un portrait que le pinceau en main. Le crayon le déroute.

Et c'est toute la capacité du peintre à pénétrer l'âme de son modèle qui rend Rolland sceptique, défaut que Thiesson partage avec d'autres $\operatorname{artistes}^{72}$.

Le 10 février, Thiesson quitte Genève et se rend à Zurich pour exposer et vendre quelques tableaux, comme il l'écrit à J.-R. Bloch, avant de conclure qu'«il a fait en Suisse quelques bons dessins et portraits, dont celui de Jouve ${ }^{73}$. Après une excursion autour du lac des Quatre Cantons et dans le canton du Tessin, les Thiesson rentrent en France sans s'arrêter à Genève, semble-t-il, pour rendre visite à Rolland. Dans une carte postée de Paris et reçue le 17 avril, le peintre lui écrit un peu sèchement:

Nous avons revu les bords de la Seine avec plaisir. Si vous m'avez écrit vous pouvez réclamer votre lettre, je n’ai pas fait suivre.

Selon toute apparence, $\mathrm{M}^{\mathrm{me}}$ Thiesson qui ne supportait plus la Suisse, a forcé son mari à rentrer en France. "Gaston regrette la Suisse, moi pas", écrit-elle à Romain Rolland ${ }^{74}$.

Dès ce moment, la correspondance change de ton et de contenu; la fréquence des lettres se fait plus irrégulière. Les préoccupations des deux hommes divergent. Le peintre se rend souvent en Bretagne pour peindre; et Rolland, qui a fini ses trois études sur Shakespeare, s'est définitivement rapproché de P. J. Jouve.

En 1916, les lettres de Rolland reflètent les principaux événements de sa vie intellectuelle: son intérêt pour la Mission Ford pour la Paix, ses rencontres avec Fried et Foerster, la nouvelle orientation de ses idées, puisqu'il a décidé de rompre le silence qu'il s'était imposé depuis juillet 1915. En revanche, il parle peu de sa nouvelle amitié avec Jouve qui écrit dans Romain Rolland vivant: «nous passâmes l'hiver ensemble, lui à

72. Lettre du 5 février 1916 à $\mathrm{M}^{\mathrm{me}}$ Rolland, C 20, p. 232 sq.

73. Lettre du 8 février 1916, C 15, p. 337.

74. Reproduit dans la lettre du 16 juin 1916 de Rolland à sa mère, C 20, p. 286. 
l'hôtel du pays, moi dans une petite maison vigneronne. " ${ }^{75}$. Alors que jusqu'à présent il voyait l'entrée en guerre des pays européens comme un "drame d'Eschyle», il veut maintenant «opposer la pure force de l'âme à la vaste force cosmique " ${ }^{76}$, ce qui aboutit selon lui à la nécessité d'un nouvel ordre social, et explique aussi son attachement à Guilbeaux et sa revue Demain. C'est dans cet esprit qu'il publie deux articles importants: «Aux peuples assassinés» et "La Route en lacets qui monte». Thiesson et Rolland ne sont plus «sur le même plan». Alors que le peintre émet dans une lettre des réticences à la lecture des poèmes de Jouve, Rolland lui écrit franchement:

[...] pour P. J. J[ouve], je proteste. Vous vous trompez, mon ami. C'est là une grande œuvre [...]. Je m'étonne que vous n'en sentiez pas la passion douloureuse ou indignée. Ce n'est pas de la tête qu'elle est sortie. C'est du cœur et des nerfs ${ }^{77}$.

Si Thiesson reproche à Jouve d'être trop cérébral, Rolland accuse maintenant Thiesson d'être trop "parisien", d'avoir les yeux fixés sur le passé, de préférer Daumier à Masereel. Il semble avoir oublié l'énergie, certes un peu vaine, avec laquelle le peintre l'a défendu ces derniers mois. Quant à Thiesson, il est le témoin du rapprochement entre Rolland et Jouve définitivement installé dans le tolstoïsme ${ }^{78}$, de cette amitié créatrice qui conduira l'hagiographe à rédiger son Romain Rolland vivant.

A la fin de l'année, Rolland écrit à sa mère qu'«il a reçu une lettre bien triste de Thiesson " ${ }^{79}$. Mesnil indique à Rolland que "sa nature sensible souffre du pitoyable spectacle de l'affaissement moral universel: son sourire si doux [lui] a semblé bien triste" ${ }^{80}$. Thiesson est tombé gravement malade. A partir de l'année suivante, les lettres se font plus rares,

75. P. J. Jouve, Romain Rolland vivant, p. 31.

76. B. Duchatelet, Romain Rolland tel qu'en lui-même, p. 196 sq. et P. J. Jouve, Romain Rolland vivant, p. 171 sq.

77. Lettre du 12 février 1918.

78. B. Bonhomme, Pierre Jean Jouve, la quête intérieure, p. 58 sq.

79. Lettre du 15 décembre 1916, C 20, p. 351, et lettre de G. Thiesson à R. Rolland du 6 décembre 1916.

8o. Lettre du 13 décembre 1916. 
tout en étant d'une fréquence régulière ${ }^{81}$. Thiesson s'intéresse encore aux activités de son ami qui désire lui faire signer son article sur «l'Indépendance de l'esprit» et l'entretient de ses déboires avec le groupe Clarté de Barbusse. Dans sa dernière lettre adressée au peintre, il écrit:

Je suis bien triste de vous savoir malade, et malade douloureusement. Combien je vous plains si votre mal ne vous laisse pas la ressource de vous en évader dans l'art ou dans la lecture ${ }^{82}$.

Thiesson meurt de la tuberculose à la maison de la santé des Frères St-Jean de Dieu, 19 rue Odinot, le 2 février 1920, là où son père était décédé. Après l'avoir rencontré la veille de sa mort, Rolland exprime une affliction vraie dans les pages de son Journal ${ }^{83}$.

Roland RoudiL Université Montpellier III

8I. Dix en 1917, 7 en 1918 (dont 3 de Thiesson). Sur les 8 lettres qui nous sont parvenues pour l'année 1919, 7 sont de la main de Rolland, ce qui laisse supposer que des lettres du peintre ont été égarées ou détruites.

82. Lettre du 30 décembre 1919.

83. Extrait du Journal de Romain Rolland, Europe, novembre-décembre, 1965, p. $198-200$. 


\section{BIBLIOGRAPHIE}

\section{Sources}

Deux hommes se rencontrent: correspondance entre Jean-Richard Bloch et Romain Rolland (1910-1918): avec une lettre de Roger Martin du Gard, Paris, Albin Michel, 1964 (Cahier Romain Rolland 15).

Fonds Romain Rolland, Bibliothèque nationale de France. Lettres inédites de R. Rolland à G. Thiesson, Madeleine Rolland, Henri Guilbeaux, Jacques Mesnil.

Jouve, Pierre Jean, Romain Rolland vivant, Paris, Ollendorff, 1920.

—, Le monde désert, Paris, Le Livre de Poche, 1968.

—, En miroir - Journal sans date, Paris, 10/18, 1972.

L'Un et l'Autre II: correspondance entre Romain Rolland et Alphonse de Châteaubriant (1914-1944), préface et annotations de L.-A. Maugendre, Paris, Albin Michel, 1996 (Cahier Romain Rolland 30).

Massis, Henri, Romain Rolland contre la France, Paris, Floury, 1915.

Rolland, Romain, De Jean-Christophe à Colas Breugnon: pages de journal, préface de Jérôme et Jean Tharaud, Paris, Editions du Salon Carré, 1946.

—, Journal des années de guerre, Paris, Albin Michel, 1950.

- Je commence à devenir dangereux: choix de lettres de Romain Rolland à sa mère (1914-1916), introduction d'Else Hartoch, Paris, Albin Michel, 1971 (Cahiers Romain Rolland 20).

Youssouri, Andicheh (éd.), Correspondance entre Romain Rolland et Gaston Thiesson (1912-1914), sous la direction de B. Duchatelet, Brest, Université de Bretagne Occidentale, Mémoire de DEA, 1990-1991. 


\section{Travaux}

Assouline, Pierre, Gaston Gallimard. Un demi-siècle d'édition française, Paris, Gallimard, 2006.

Bonhomme, Béatrice, Pierre Jean Jouve, la quête intérieure, Paris, Adens, 2008.

Duchatelet, Bernard, Romain Rolland tel qu'en lui-même, Paris, Albin Michel, 2002.

Rameix, Christophe, L'Ecole de Crozant. Les peintres de la Creuse et de Gargilesse 1850-1950, Saint-Paul, Lucien Souny, 2002.

SiCard, Claude (éd.), Correspondance Jacques Copeau-Roger Martin du Gard, t. II, Paris, Gallimard, 1972.

Stelling-Michaud, Sven, "Romain Rolland, Edmond Rossier et la Bibliothèque Universelle», Cahiers Vilfredo Pareto, 22-23 (1970), p. 109-121.

White, Palmer, Poiret le magnifique. Le destin d'un grand couturier, Paris, Payot, 1986. 